\title{
On the problem of Plasma Sheet Boundary Layer identification from plasma moments in Earth's magnetotail
}

\author{
E. E. Grigorenko ${ }^{1}$, R. Koleva ${ }^{2}$, and J.-A. Sauvaud ${ }^{3}$ \\ ${ }^{1}$ Space Research Institute of RAS, Moscow, Russia \\ ${ }^{2}$ Space Research and Technologies Institute of BAS, Sofia, Bulgaria \\ ${ }^{3}$ Institute de Recherche en Astrophysique et Planétologie, Toulouse, France \\ Correspondence to: E. E. Grigorenko (elenagrigorenko2003@yahoo.com)
}

Received: 28 December 2011 - Revised: 14 August 2012 - Accepted: 19 August 2012 - Published: 11 September 2012

\begin{abstract}
The problem of identification of the interface region between the lobe and the Plasma Sheet (PS) - the Plasma Sheet Boundary Layer (PSBL) - using ion moments and magnetic field data often arises in works devoted to statistical studies of various PSBL phenomena. Our experience in the identification of this region based on the analysis of ion velocity distribution functions demonstrated that plasma parameters, such as the ion density and bulk velocity, the plasma beta or the dynamic pressure vary widely depending on the state of magnetotail activity. For example, while field-aligned beams of accelerated ions are often observed propagating along the lobeward edge of the PSBL there are times when no signatures of these beams could be observed. In the last case, a spacecraft moving from the lobe region to the PS registers almost isotropic PS-like ion velocity distribution. Such events may be classified as observations of the outer PS region. In this paper, we attempt to identify ion parameter ranges or their combinations that result in a clear distinction between the lobe, the PSBL and the adjacent PS or the outer PS regions. For this we used 100 crossings of the lobe-PSBL-PS regions by Cluster spacecraft $(\mathrm{s} / \mathrm{c})$ made in different periods of magnetotail activity. By eye inspection of the ion distribution functions we first identify and separate the lobe, the PSBL and the adjacent PS or outer PS regions and then perform a statistical study of plasma and magnetic field parameters in these regions. We found that the best results in the identification of the lobe-PSBL boundary are reached when one uses plasma moments, namely the ion bulk velocity and density calculated not for the entire energy range, but for the energies higher than $2 \mathrm{keV}$. In addition, we demonstrate that in many cases the plasma beta fails to cor-
\end{abstract}

rectly identify and separate the PSBL and the adjacent PS or the outer PS regions.

Keywords. Magnetospheric physics (Magnetotail)

\section{Introduction}

The plasma sheet boundary layer (PSBL) is the layer at the lobe-plasma sheet (PS) interface. Yet there is no unanimous definition of the PSBL. It is widely accepted that the most important feature of the PSBL is the observation of field-aligned beams of accelerated ions reported by numerous researches (e.g., DeCoster and Frank, 1979; Forbes et al., 1981; Eastman et al., 1984; Takahashi and Hones, 1988; Parks et al., 1998). The PSBL spatial structure, described by Takahashi and Hones (1988), gives the classical view of this region. When a satellite moves from the lobe to the PS, crossing the lobe/PS separatrix, one first encounters a hot electron boundary, then high energy field aligned earthward ion beams, then counterstreaming ion beams and after that the more or less isotropic PS ions are observed.

If we consider the PSBL as the separatrix layer between the open lobe field lines and the closed field lines of the plasma sheet, at least three states of the lobe-PS interface could be identified. Grigorenko et al. (2009) reported two "extreme" states of the PSBL observed during different periods of geomagnetic activity.

The first one, typically observed during quiet periods, is characterised by observations of long-lasting energy collimated field-aligned ion beams with energies below $40 \mathrm{keV}$. These beams (the so called Type-I beams) are accompanied by electrons having almost isotropic velocity distribution 
function, which are observed even at the lobe side of the ion beams, indicating that ion acceleration occurs in the distant Current Sheet (CS) at closed magnetic field lines. During these periods no clear magnetic separatrix between yet open and already closed magnetic field lines is observed in the electron velocity distribution function. Strictly speaking, for such events one may identify only the "ion PSBL" boundary, i.e., the boundary between the region where ions with energies higher than $2 \mathrm{keV}$ are absent and the region where they are observed.

The second PSBL state is characterised by the occurrence of more energetic, up to $\sim 200 \mathrm{keV}$ ion beams (the so called Type-II ion beams), with broader distribution in parallel velocities. These ion beams are accompanied by electrons with anisotropic along the magnetic field velocity distribution functions, comprised of cold electron beams $(<1 \mathrm{keV})$ moving towards an acceleration source (usually tailward of Cluster s/c location) and by hot electrons $(\geq 1 \mathrm{keV})$ streaming from the acceleration source (i.e., earthward of Cluster $\mathrm{s} / \mathrm{c}$ location). This feature indicates crossing of the magnetic separatrix between still open and already reconnected field lines and implies that the ion beams are accelerated near the magnetic reconnection region.

Finally, there is a third state of the lobe-PS interface which is characterised by the absence of field-aligned ion beams. During such intervals a spacecraft, moving from the lobe region (or the region empty of $\mathrm{keV}$ ions), starts to register at once almost isotropic PS-like velocity distribution function of $\mathrm{keV}$ ions without any signature of ion beam. Many authors consider this an absence of a PSBL, which could be due to the absence of ion acceleration in the CS during such periods. Angelopoulos et al. (1993) called this state "quasi-static plasma sheet boundary" and they assumed the layer at the PS boundary to be an outer plasma sheet. On the contrary, Baumjohann et al. (1988) pointed out that though no ion beams exist, a well-defined layer - a PSBL, with stable characteristics, different from those of the PS, always exists at the lobe-PS interface. Multipoint observations shed a new light on this problem. Grigorenko et al. (2007), Grigorenko and Koleva (2009), analysing several cases of PSBL crossings by the Cluster quartet, showed that the lobe-PS interface sometimes is not uniform in the dawn-dusk direction according to the features of ion velocity distribution functions: in this region flux tubes of finite cross-sections, containing either accelerated field-aligned ion beams or almost isotropic PS-like plasma simultaneously exist. Due to the large-scale fluctuations propagating earthward these tubes successively crossed Cluster s/c. A possible explanation of the absence of ion beam observation, proposed by Grigorenko et al. (2012), is related to the spatial localisation of the acceleration source(s) in the dawn-dusk direction.

Statistical studies of various PSBL phenomena often require an analysis of a huge volume of data. Therefore, many researchers try to find some algorithm for an automatic search of this region using plasma and magnetic field data measured in the magnetotail along a spacecraft orbit without visual inspection of ion velocity distribution functions. A widely used criterion is the $\mathrm{X}$-component of ion velocity, which in the lobe-PSBL interface is roughly parallel to the magnetic field while in the Central Plasma Sheet (CPS) it is roughly perpendicular to the local magnetic field. Indeed, if a spacecraft is located in the PSBL, an enhancement of $V_{X}$ (GSE) component up to several hundreds of $\mathrm{km} \mathrm{s}^{-1}$ is due to field-aligned ion beam propagation. However, it could be due to convective flow propagation, for example, a BBF, if the spacecraft is located in the CPS (e.g., Nakamura et al., 1991; Angelopoulos et al., 1992, 1994; Petrukovich et al., 2001). Therefore, by using only this parameter it is impossible to define in which region a spacecraft is located.

Some advantage in solving this problem could be reached if one uses the values of the X-component of the ion fieldaligned velocity $V_{\| X}$ to identify the PSBL region and the Xcomponent of the perpendicular velocity to identify the CPS region with convective flows (e.g., Angelopoulos et al., 1994; Nagai and Machida, 1998). However, another problem arises here: what is the threshold for $V_{\| X}$ values, which, as we will show below, may vary in the PSBL in a rather wide range. Moreover, parallel ion flows or beams are often registered not only in the PSBL, but also in the PS (e.g., Raj et al., 2002) region.

Because of these difficulties many researchers have used the value of plasma beta $(\beta)$, which represents the ratio of the thermal plasma pressure to the magnetic pressure. As it was reported in previous statistical studies, the typical values of plasma beta in the PSBL region are usually below 0.1 and in the PS and the CPS it is usually $\geq 1.0$ (e.g., Baumjohann et al., 1988, 1989). In the paper by Baumjohann et al. (1988) the authors tried to identify the intervals of the PSBL crossings using plasma $\beta$, magnetic field value, its deviation from the Sun-Earth line and the value of the photoelectron flux. They reported significant overlapping of these parameters measured in the PSBL and in the adjacent PS region, especially for the plasma $\beta$. Angelopoulos et al. (1994), on the contrary, made an attempt to identify the CPS region with fast convective flows and to exclude from consideration the PSBL region. For the identification of CPS the authors used the following criterion to search the CPS region with fast convective flows: $\left|V_{X}\right|>400 \mathrm{~km} \mathrm{~s}^{-1}$ and $\beta>0.5$. However, the statistical study performed by Raj et al. (2002) demonstrated that the separation of the PSBL region with field-aligned ion beams and the PS with bulk flows using only moment-based parameters or thresholds leads to significant errors. Nevertheless, they suggested as optimal criteria for the selection of bulk flow events: $V_{\perp}>250 \mathrm{~km} \mathrm{~s}^{-1}$ and plasma $\beta_{X Y}$ (based on the $\mathrm{X}$ - and Y-components of the magnetic field) $>2$.

In this work, we concentrate on the problem of identification of the PSBL-lobe and the PSBL-PS boundaries using Cluster data. The structure of our paper is the following. In the second section we describe the data and method used in this study. In the third section we present a couple of case 
studies, typical for different periods of magnetotail activity. This is done in order to demonstrate how much ion moments measured in the lobe-PSBL-PS regions may vary in time, and how the observation pattern deduced from plasma moments may differ from the one obtained by an analysis of ion velocity distribution functions. In the fourth section we present a statistical study of different plasma parameters measured in the lobe, the PSBL and the adjacent PS or outer PS regions, which were predetermined by an eye inspection of ion velocity distribution functions. We show the typical ranges of ion density, bulk velocity, plasma $\beta$ and ion dynamic pressure measured in these three regions and the extent of their overlapping. Then we compare the values of these parameters calculated for the entire ion energy range and for its highenergy part $(>2 \mathrm{keV})$. We demonstrate that the use of these moments decreases the error in the PSBL-lobe and PSBLPS separations. Finally in the last section, we discuss the obtained results and the errors, which one should take into acount while trying to distinguish the lobe-PSBL-PS interface regions by using moment-based selection criteria only.

\section{Data analysis}

The premise of this study is the ion data observed by all ion instruments in the energy range from $20 \mathrm{eV}$ to $40 \mathrm{keV}$. In this paper we repeat essentially the analysis performed by Raj et al. (2002) as we also combine a statistical study based on automatic search for the PSBL region using different plasma parameters with careful eye inspection of ion velocity distribution functions observed in each of the analysed intervals of the lobe-PSBL-PS crossings by Cluster s/c. We pay special attention to the identification of PSBL-lobe and PSBLPS boundaries.

Unlike Raj et al. (2002), in our analysis we also used ion moments calculated for the high energy part of the spectrum (for energies higher than $2 \mathrm{keV}$ ). We used this energy threshold in order to exclude the influence of ionospheric ions, especially $\mathrm{O}^{+}$. As was reported by, for example, Sauvaud et al. (2004) the energy of initially cold ionospheric ions moving tailward near the lobeward edge of the PSBL may increase up to $1-2 \mathrm{keV}$ due to the increase of their drift velocity. Such an increase could be caused either by the increase of the dawn-dusk electric field (e.g., Mukai et al., 1994) or by large scale electromagnetic fluctuations propagating along the PSBL magnetic flux tubes (e.g., Grigorenko et al., 2010).

For our study we used measurements of three-dimensional ion velocity distribution functions and moments collected every $12 \mathrm{~s}$ (two-dimensional (2-D) distribution is collected for $62.5 \mathrm{~ms}$ ) by the Hot Ion Analysers (HIA) onboard Cluster-1 and -3 (Rème et al., 2001). For case studies we also used proton data measured by the time-of-flight ion Composition and Distribution Function analyser (CODIF) onboard Cluster-4 (Rème et al., 2001) and electron measurements provided by the Plasma Electron and Current Experiment (PEACE, Owen et al., 2001) onboard Cluster-1 and obtained from the Cluster Active Archive (CAA). The magnetic field data were obtained with a 4-s resolution by the FluxGate Magnetometer (FGM, Balogh et al., 2001). Geomagnetic indices (AL) were retrieved from the World Data Center for Geomagnetism, Kyoto (http://wdc.kugi.kyoto-u.ac.jp).

First we identified and separated the intervals of crossings of the lobe, the PSBL and the adjacent PS or the outer PS regions by careful eye inspection of ion velocity distribution functions and then performed a statistical study of the plasma and the magnetic field parameters in these regions. We define the lobe region as the region where ions with energies higher than $2 \mathrm{keV}$ are absent (the number density of these ions is less than $0.01 \mathrm{~cm}^{-3}$ ). However, this definition is correct only for periods of Type-II ion beam propagation in the PSBL. Only during such periods the boundary of "ion PSBL" coincides (accurate within time resolution) with the magnetic separatrix between open and closed magnetic field lines. During the periods of Type-I ion beam propagation or the periods of quasi-static PS boundary observation it is particularly difficult to identify exactly the lobeward edge of the PSBL or outer PS, taking in mind the definition of the lobe, as the region with open magnetic field lines. The region located just lobeward of the Type-I ion beam (or quasi-static PS boundary) is still the region of closed magnetic topology since isotropic electron velocity distribution functions are registered there. Thus, an identification of the actual boundary between open and closed field lines is impossible by using only ion moments without careful inspection of electron velocity distribution functions.

Grigorenko et al. (2009) used the skewness parameter to analyse the asymmetry of 1-D cuts of the electron velocity distributions along the magnetic field in order to check the presence of the magnetic separatrix. However, the development of an automatic search based on such algorithm is beyond the scope of the present paper. In this work, under "lobeward edge of PSBL" we mean only the lobeward boundary of "ion PSBL" and not the actual PSBL boundary.

A region that we classify as the PSBL should satisfy the following criteria:

1. for ion energies higher than $2 \mathrm{keV}$, the maximum of velocity distribution function should be observed in the angular sector corresponding to the field-aligned direction;

2. the ratio of the phase density in the sectors, corresponding to the direction perpendicular to the local magnetic field, to the phase density in the field-aligned sector (which corresponds to the maximum of velocity distribution function) should be less than 0.3 .

The PS region adjacent to the PSBL, or the region adjacent to the quasi-static PS boundary at which no accelerated field-aligned beams are observed, could be considered as the outer PS. This region is classified as the region which does 


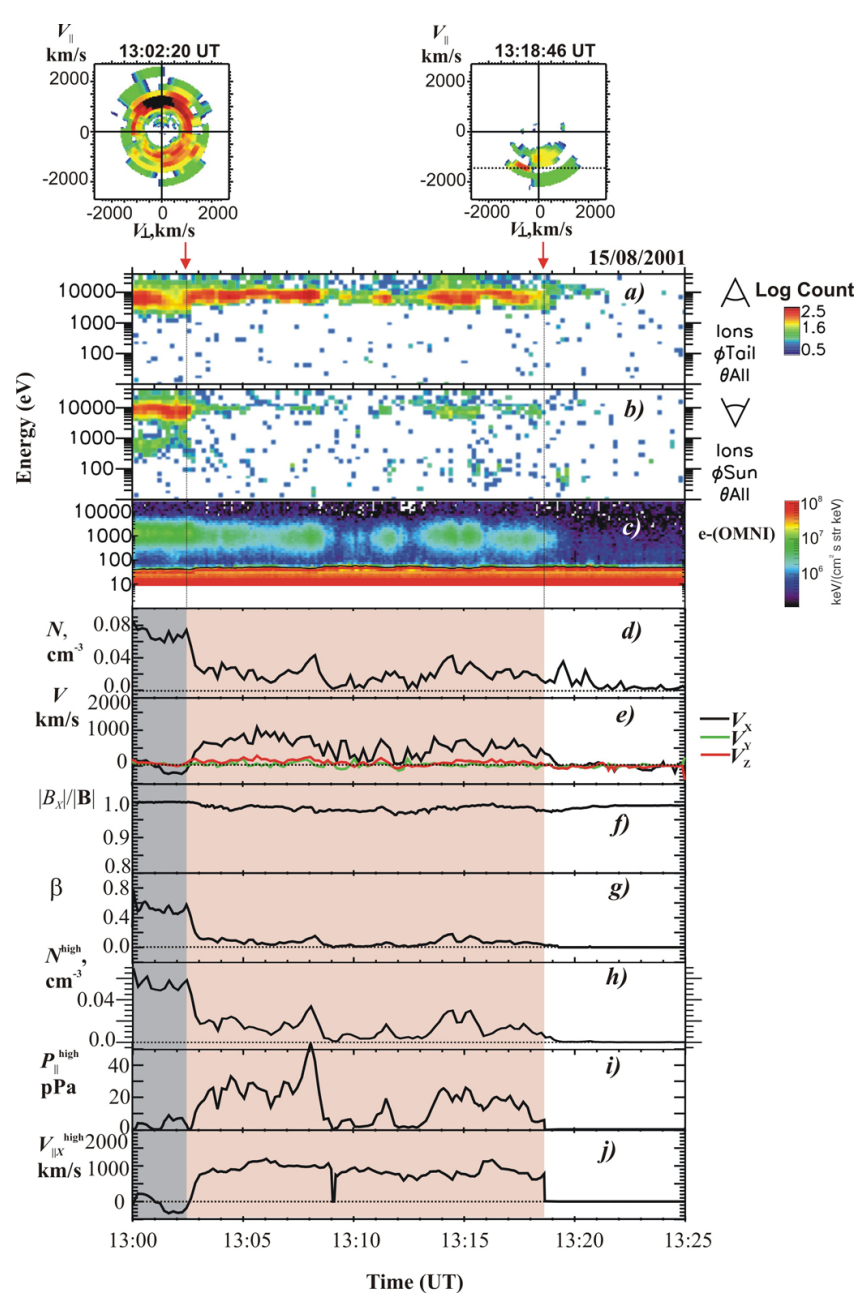

Fig. 1. PS-PSBL-lobe crossing by Cluster s/c on 15 August 2001 between 13:00-13:25 UT. Ion data were measured by the HIA instrument onboard Cluster-1. Electron data were detected by the PEACE experiment onboard Cluster-2. In the upper part of the Fig. 2d cuts of ion velocity distribution functions plotted in the $\left(V_{\perp}, V_{||}\right)$ plane are shown. Below, from top to bottom: $(\mathbf{a}, \mathbf{b})$ Energy-Time $(E-T)$ spectrograms of earthward and tailward moving ions correspondingly; (c) $E-T$ spectrogram of omni-directional electrons; (d, e) ion density $N$ and three GSE components of velocity calculated for the entire energy range; (f) time profile of the value $\left|B_{X}\right| /|\mathbf{B}|$; (g) plasma $\beta$; $(\mathbf{h}-\mathbf{j})$ ion density $N^{\text {high }}$, parallel dynamic pressure $P_{\|}^{\text {high }}$ and X-component of the parallel velocity $V_{\| X}^{\text {high }}$ calculated for the high-energy part of the spectrum $(>2 \mathrm{keV})$.

not satisfy, at least, the second PSBL criterion. It is worthy to note that the boundary of the quasi-static PS (where no accelerated ion beams are observed) cannot be distinguished from the PS region adjacent to the PSBL. Thus, in order to avoid confusion, throughout the paper we will consider both these regions as the outer PS region.

\section{Observations in the lobe-PSBL-PS interface during different periods of magnetotail dynamics}

Below we present two intervals of the lobe-PSBL-PS crossings by Cluster s/c which took place during different periods of magnetotail activity. The first event is shown in Fig. 1. On 15 August 2001 within 13:00-13:25 UT Cluster s/c was located in the Southern Hemisphere at $[-16.9,-3.6,-4.6]$ $R_{\mathrm{E}}$ (GSE) and moved from the PS towards the lobe region crossing the PSBL. Ion and electron data presented in Fig. 1 were obtained by Cluster-1. The ion and electron observations provided by Cluster- 3 and Cluster- 4 are similar to the ones presented in Fig. 1 and not shown here. During this interval geomagnetic activity was very low: the absolute value of the AL index was below $100 \mathrm{nT}$.

As the spacecraft exits from the PS (in Fig. 1 the interval corresponding to the observation of PS-like ion velocity distribution functions is shaded by grey) to the PSBL, a field-aligned beam of accelerated ions started to be observed. As it is seen from the 2-D cuts of the ion velocity distribution functions plotted in $\left(V_{\perp}, V_{\|}\right)$plane and shown above the upper panel of Fig. 1, beam ions moved earthward and had parallel velocity of about $-1500 \mathrm{~km} \mathrm{~s}^{-1}$. An analysis of ion velocity distribution functions revealed that this ion beam was observed during $\sim 16.5 \mathrm{~min}$ (the corresponding interval is shaded in pink) and was accompanied by electrons having velocity distribution functions almost isotropic not only inside the PSBL, but also on the lobe side of the ion beam (not shown in the figure). This ion beam can be classified as Type-I ion beam, which was accelerated in the distant Current Sheet (CS) source located at closed magnetic field lines (Grigorenko et al., 2009).

During the entire interval the ratio of $\left|B_{X}\right| /|\mathbf{B}|$ was close to 1.0 (see Fig. 1f) even when the Cluster s/c was in the PS. We should note here that the interval of interest does not contain the crossing of CPS where $\left|B_{X}\right| /|\mathbf{B}|$ is significantly smaller than 1.0, since our task is to separate the PSBL from the lobe and the outer PS region. It is worthy to note that for this event the value of $\left|B_{X}\right| /|\mathbf{B}|$ is inadequate for such separation.

The ion density shown in panel d of Fig. 1 was calculated for the entire energy range $(\sim 5 \mathrm{eV}-32 \mathrm{keV})$ of HIA instrument onboard Cluster-1. It is seen that the ion density does not decrease significantly, but fluctuates at the lobeward edge of the ion PSBL which was identified as the last observation of statistically valid field-aligned velocity distributions of accelerated ions. Such behaviour of density value could be due to the presence of low-energy ion population in the adjacent high-latitude region.

The plasma $\beta$ also did not experience a significant variation at the lobe side of the ion beam: its value was about 0.05 inside the PSBL (Fig. 1g). The X-component of ion velocity, calculated for the entire energy range, varies within the PSBL from almost zero values (at times when counterstreaming ion beams are observed or at times when the s/c approaches the lobe, probably because of tail flapping) to $\sim 1000 \mathrm{~km} \mathrm{~s}^{-1}$ and 


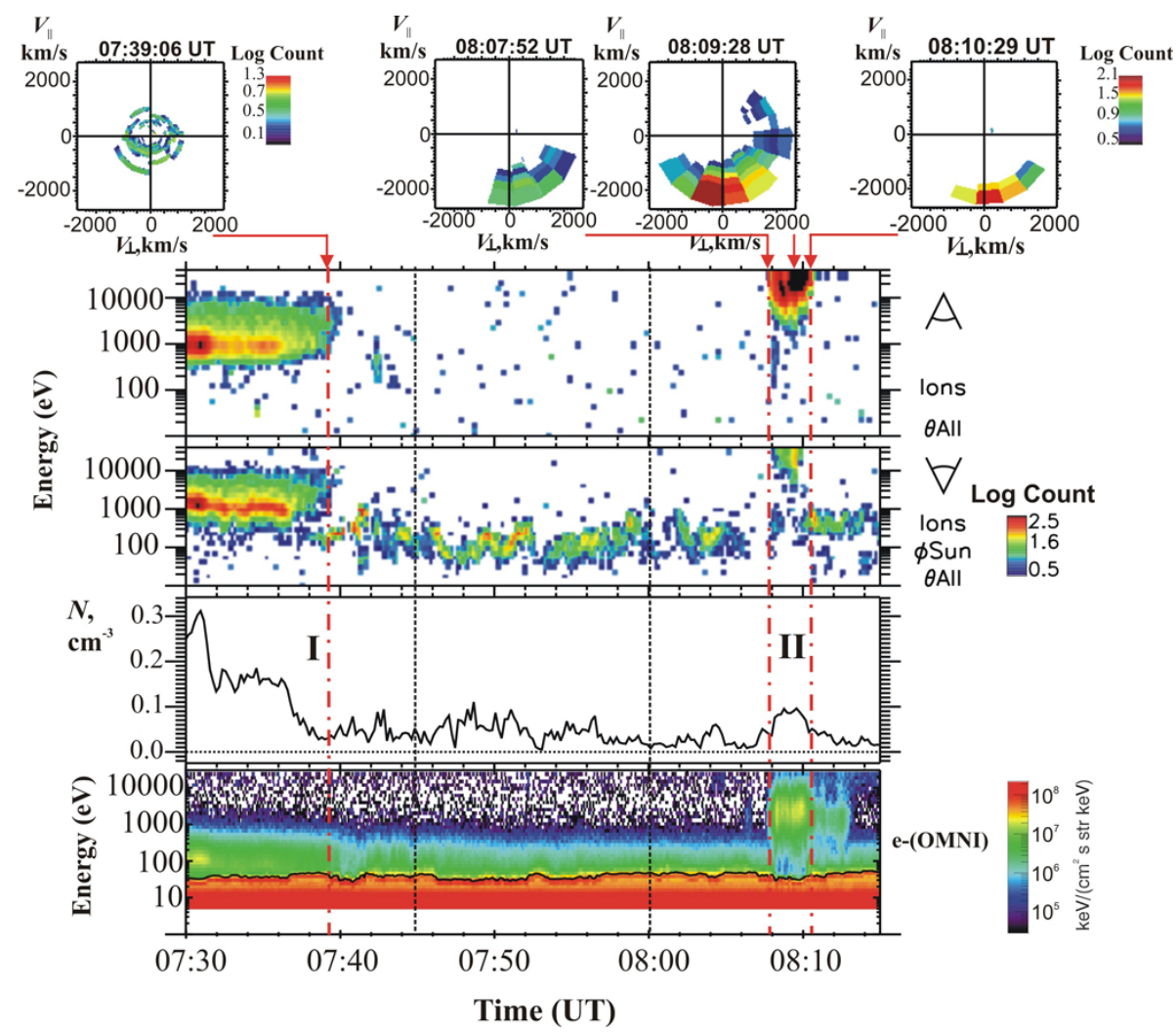

Fig. 2. PS-lobe interface crossing and PSBL encounter observed on 22 September 2001 by Cluster s/c. Ion data presented in the figure where measured by the HIA instrument onboard Cluster-1. Electron data were detected by the PEACE experiment also onboard Cluster-1. In the upper part of the Fig. $2 \mathrm{~d}$ cuts of the ion velocity distribution functions plotted in the $\left(V_{\perp}, V_{\| \mid}\right)$plane are shown. Below, from top to bottom: $E-T$ spectrograms of earthward and tailward moving ions and ion density $N$, calculated from the entire energy; bottom panel shows $E-T$ spectrogram of omni-directional electrons.

it gradually decreases to zero value as the Cluster s/c exits from the ion PSBL to the high-latitude region (Fig. 1e). Thus, among the listed parameters $V_{X}$ is more useful for the identification of the lobeward boundary of ion PSBL if it contains an ion beam.

The three bottom panels of Fig. 1 show the ion density $N^{\text {high }}$, the X-component of ion parallel velocity, $V_{\| X}^{\text {high }}$, and the ion dynamic pressure $P_{\|}^{\text {high }}=m_{\mathrm{p}} N^{\text {high }}\left(V_{\|}^{\text {high }}\right)^{2}$, calculated for the high energy part of the ion spectrum (for energies higher than $2 \mathrm{keV}) . V_{\| X}^{\text {high }}$ and $P_{\|}^{\text {high }}$ were put to zero if the density of the high energy ions was below $0.01 \mathrm{~cm}^{-3}$. Thus, these parameters have zero values at the region located at higher latitudes than the ion PSBL, and in the lobe region, that immediately allows their separation from the PSBL and PS regions. It is seen that the values of $V_{\| X}^{\text {high }}$ and $P_{\|}^{\text {high }}$ experienced jumps at the lobeward edge of ion PSBL: the value of $V_{\| X}^{\text {high }}$ increases up to $800 \mathrm{~km} \mathrm{~s}^{-1}$ and $P_{\|}^{\text {high }}$ - up to $\sim 7 \mathrm{pPa}$ in comparison with their zero values in the adjacent high-latitude region. The PSBL region, namely its part where only field-aligned ion beam streaming in one direction is observed, could be identified according to the value of ion $V_{\| X}^{\text {high }}$ : for example, in this event it ranges from 500 to $1200 \mathrm{~km} \mathrm{~s}^{-1}$. In the part of the PSBL closest to the PS and where a significant flux of counterstreaming field-aligned ions (directed tailward in this event) is registered, the value of $V_{\| X}^{\text {high }}$ decreased to negative values. We consider that this part of PSBL and especially its boundary with the PS are almost impossible to identify from ion moments. Only a visual analysis of ion velocity distribution functions allows this identification.

Another interval of interest -22 September 2001 between 07:30-08:15 UT ( $|\mathrm{AL}| \sim 200 \mathrm{nT})-$ is shown in Fig. 2. Cluster was located in the Southern Hemisphere at $[-17.9,6.3$, $-0.9] R_{\mathrm{E}}$ (GSE) and at the beginning of this interval, between 07:30-07:45 UT, it exited the PS and entered the highlatitude region empty of high energy ions without observation of field-aligned ion beam. Again, ion and electron data presented in the figure were obtained by Cluster- 1 . The time of crossing the lobeward edge of the outer PS is marked "I" in Fig. 2. We identified the region observed before the time "I" as the outer PS, because of the almost isotropic ion velocity distribution functions whose characteristics satisfied our PS 
a)

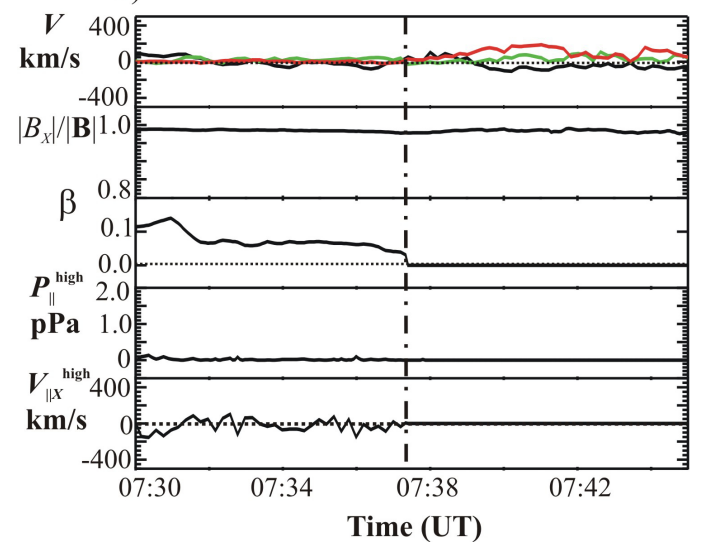

b)

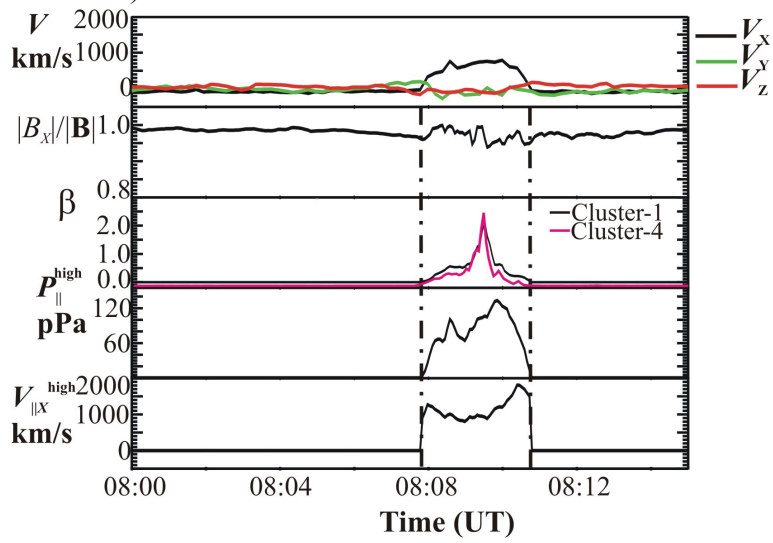

Fig. 3. Zoom of plasma and magnetic field parameters observed by Cluster-1 during intervals of (a) PS-lobe and (b) PSBL crossings on 22 September 2001. From top to bottom: three components of ion velocity (in GSE) calculated for the entire energy range; time profile of $\left|B_{X}\right| /|\mathbf{B}|$, plasma $\beta$, parallel dynamic pressure $P_{\|}^{\text {high }}$ and X-component of the ion parallel velocity, calculated for the high energy part of the spectrum $(>2 \mathrm{keV})$. Additionally in (b) the plasma beta calculated from proton data measured by the CODIF instrument onboard Cluster-4 is shown. The times of crossings of the lobeward boundaries of the PS and the PSBL are shown by the dot-and-dashed lines.

criterion. As well as in the previous event, we did not discuss here the interval of the CPS crossing.

The zoom of the interval between 07:30-07:45 UT is shown in Fig. 3a. It is seen that both in the outer PS and in the adjacent high-latitude region the value of $\left|B_{X}\right| /|\mathbf{B}|$ ratio was close to 1.0 and almost did not change when the spacecraft had exited the outer PS and entered the high-latitude region. Due to the absence of accelerated ion beams or parallel plasma flows, no increases of $V_{\| X}^{\text {high }}$ and $P_{\|}^{\text {high }}$ were observed. The only parameter, which experiences a significant increase in the PS region, was the plasma $\beta$. While in the adjacent high-latitude region it was almost zero, at the high-latitude boundary of the outer PS it increases up to $\sim 0.04$ and keeps increasing deeper in the PS. However, as we show below in the statistical analysis of different periods of magnetotail activity, larger $\beta$ values were observed in the PSBL, especially during periods of powerful ion acceleration in the magnetotail.

To confirm this let us consider another part of the interval shown in Fig. 2, between 08:00-08:15 UT. This interval contains a short encounter (between $\sim 08: 08-08: 10: 50$ UT) with the PSBL, which was registered by all Cluster satellites. Actually, this encounter is the first from a series which took place during the next two hours due to transient PS expansions. During this short PSBL encounter, indicated in Fig. 2 by the label "II", two crossings of the lobeward edge of the PSBL took place along with the observation of strong earthward moving field-aligned ion beam. In this event the parallel velocity of the ion beam reached $-2500 \mathrm{~km} \mathrm{~s}^{-1}$ (see the ion velocity distribution functions shown in the upper part of Fig. 2). Electrons observed during this time in the PSBL have temperature higher than $1 \mathrm{keV}$ and their velocity distribution functions registered near the lobeward edges of the PSBL are anisotropic and consist of accelerated electron flux moving in the same direction as the ion beam (i.e., earthward) and electrons with lower energies moving towards the acceleration source (tailward) (not shown in the figure). Similar features in the electron velocity distribution functions measured at the lobeward edge of the PSBL were reported earlier by Nagai et al. (2001), Fujimoto et al. (2001), Nakamura et al. (2004) and were associated with the spacecraft crossing of the magnetic separatrix between the yet open and already reconnected magnetic field lines. Thus, the simultaneously observed field-aligned ion beam (the so-called Type-II ion beam) was most likely accelerated near the magnetic reconnection region, located tailward from the Cluster s/c.

The zoom of this interval is shown in Fig. $3 b$. It is seen that during this PSBL crossing the $V_{X}$ component of the ion velocity, calculated from the entire energy range, increased at the lobeward edge of the PSBL only up to $400 \mathrm{~km} \mathrm{~s}^{-1}$, while the X-component of the ion parallel velocity, $V_{\| X}^{\text {high }}$, increased up to $900 \mathrm{~km} \mathrm{~s}^{-1}$ and ranged from 900 to $1800 \mathrm{~km} \mathrm{~s}^{-1}$ inside the PSBL. Correspondingly, the dynamic pressure $P_{\|}^{\text {high }}$ also experienced significant enhancement during this crossing up to $\sim 135 \mathrm{pPa}$. It is worth to note that the plasma $\beta$ was also large in the PSBL: it was approximately 0.3 near its lobeward boundary, which is already larger than it was observed during the previous PS crossing. Deeper in the PSBL the plasma $\beta$ became larger and reached 2.2 according to the Cluster- 1 data and $\sim 2.5$ according to the proton data obtained by the CODIF instrument onboard Cluster-4, the value more typical for the CPS (e.g., Baumjohann et al., 1989).

Thus, one can observe a significant overlapping of plasma parameters measured in the outer PS and in the PSBL just within $1 \mathrm{~h}$. In the next section, we confirm this by statistical analysis of the plasma parameters, observed in the lobe, 


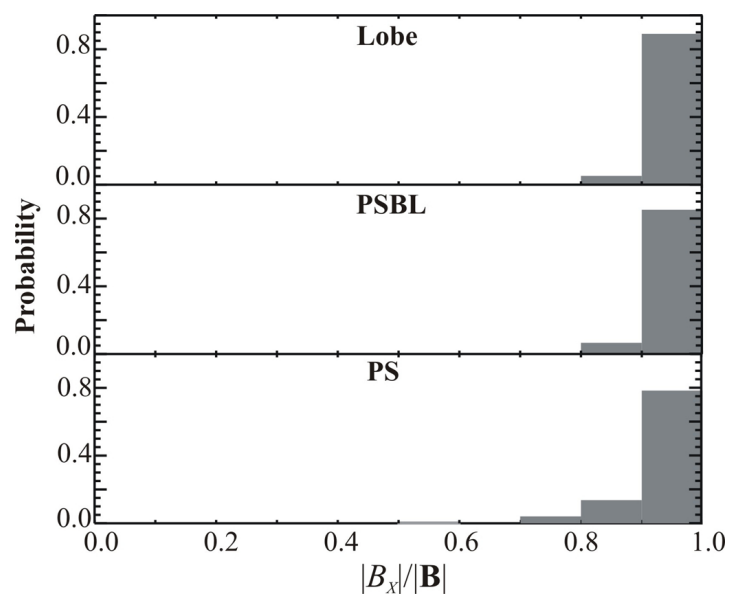

Fig. 4. Histograms of the distribution of the observation probability of $\left|B_{X}\right| /|\mathbf{B}|$ values in the lobe, the PSBL and the outer PS regions.

the PSBL and the outer PS region during different periods of magnetotail activity. We try to select among the analysed parameters those that provide smaller error in the separation between these three regions.

\section{Statistical study of plasma parameters observed in the lobe, the PSBL and the PS regions during different periods of magnetotail dynamics}

For the statistical study of the typical plasma parameters observed in the lobe, the PSBL and the outer PS regions we used intervals of crossings of these regions by Cluster s/c during its magnetotail sessions in August-October of 2001. Only intervals when Cluster s/c had crossed magnetotail at $X<-15 R_{\mathrm{E}}$ were considered. Our analysis includes 12446 12-s bins of ion measurements and 37338 bins of 4-s magnetic field measurements. Among them, visual inspection of the ion velocity distribution functions allowed the classification of 3353 bins (10059 magnetic field bins) as belonging to the lobe region, 5071 bins (15213 magnetic field bins) as belonging to the PSBL region and 4022 bins (12066 magnetic field bins) belonging to the outer PS region. In the present study we do not include intervals of CPS crossings. Samples characterised by isotropic ion velocity distribution functions and by $\left|B_{X}\right| \leq 10 \mathrm{nT}$ are identified as CPS samples and are excluded. Samples characterised by almost isotropic ion velocity distribution functions (the second criterion of the PSBL definition is not fulfilled) and by $\left|B_{X}\right|>10 \mathrm{nT}$ are considered to be outer PS samples.

Figure 4 shows histograms of the distribution of the observation probability of $\left|B_{X}\right| /|\mathbf{B}|$ values in the three analysed regions: the lobe, the PSBL and the outer PS. It is seen from the figure that there is a large overlapping of this parameter in the three regions: the most frequently observed values of $\left|B_{X}\right| /|\mathbf{B}|$ range from 0.8 to 1.0 . In the outer PS region where

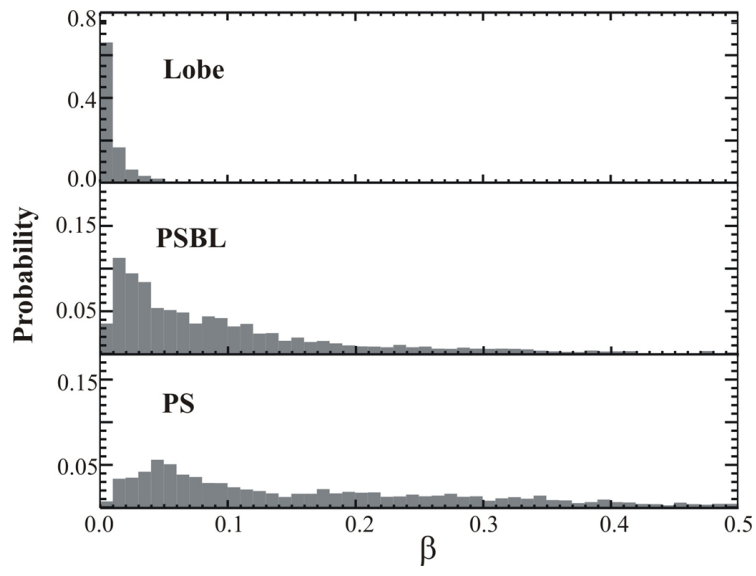

Fig. 5. Histograms of the distribution of the observation probability of the plasma $\beta$ in the lobe, PSBL and the outer PS regions calculated for the most frequently observed $\beta$ range.

the ion velocity distribution functions are already isotropic the value of $\left|B_{X}\right| /|\mathbf{B}|$ exceeds 0.8 in almost $80 \%$ of the analysed cases. Thus the use of $\left|B_{X}\right| /|B|$ parameter fails to distinguish the PSBL from the adjacent lobe and the outer PS regions.

Histograms of the distributions of the observation probability of the plasma $\beta$ in the lobe, the PSBL and the outer PS regions are shown in Fig. 5. The range of $\beta$ values displayed in the figure corresponds to the most frequently observed $\beta$ values $(\leq 0.5)$. It is seen that the distributions for the PSBL and the outer PS regions have long "tails" (for $\beta>0.1$ ). In order to show the entire range of $\beta$ values observed in the PSBL and the outer PS regions in Fig. 6 we present a scatterplot of $\beta$ values versus the values of the ion density, calculated for the entire energy range and observed in the PSBL (blue dots) and in the outer PS region (red dots). It is worth to note that although the scatterplot presented in Fig. 6 displays many PSBL samples with $\beta>0.5$ the probability to observe these $\beta$ values in the PSBL region does not exceed few percents.

From Figs. 5 and 6 one can see the overlap in $\beta$ values measured in different regions: ion $\beta \leq 0.01$, which is typical for the lobe region (65\% of the cases), was registered in $\sim 3.5 \%$ of the PSBL and in $1 \%$ of the outer PS samples. The maximum of $\beta$ distribution measured in the PSBL corresponds to $\beta$ range from 0.01 to 0.02 . Approximately in $17 \%$ of the lobe samples the measured ion $\beta$ also has values of $0.01-0.02$.

The separation between the PSBL and the outer PS region according to the plasma $\beta$ value is more difficult. It is seen that $\beta$ values within the range of $0.05-0.2$ are observed both in the PSBL and in the outer PS with approximately equal probability. Although as a whole the outer PS samples are characterised by higher $\beta$ values than the PSBL samples, statistically the PSBL samples and the samples belonging to the 


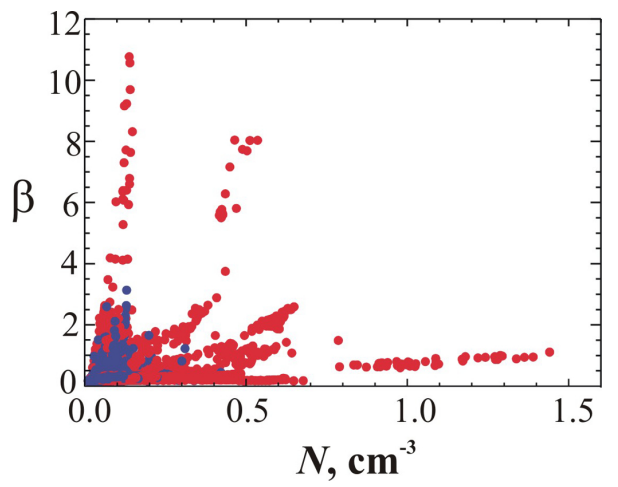

Fig. 6. Scatterplot of the plasma $\beta$ versus the ion density calculated for the entire energy range. The samples measured within the PSBL are shown by blue dots, and those measured in the outer PS region are displayed by red dots.

intervals of the cold outer PS crossings are hard to be separated using only the $\beta$ parameter.

We also try to use the ion dynamic pressure $P_{\|}^{\text {high }}$, calculated for the high energy part of the spectrum $(>2 \mathrm{keV})$. Figure 7 presents histograms of the distribution of the observation probability of $P_{\|}^{\text {high }}$ in the PSBL and the outer PS region. Again one can see a significant overlapping of the values observed in both regions: in approximately $46 \%$ of PSBL samples and in $81 \%$ of outer PS samples the observed values of $P_{\|}^{\text {high }}$ are below $5 \mathrm{pPa}$.

Figure 8 presents histograms of the distribution of the observation probability of the absolute value of the $\mathrm{X}$ component of the ion parallel velocity, $V_{|| X}$, calculated for the entire energy range (a) in the lobe, the PSBL and the outer PS regions and (b) for the high energy part of the spectrum $(>2 \mathrm{keV})$ for the PSBL and the outer PS regions. It is immediately seen from Fig. 8a that the use of $V_{|| X}$ is not good enough for the separation between these three regions: the maximum of all distributions corresponds to $V_{\| X} \leq$ $100 \mathrm{~km} \mathrm{~s}^{-1}$. In the lobe region such distribution is produced by ions of ionospheric origin, which move tailward along the magnetic field lines with velocities of $\leq 100 \mathrm{~km} \mathrm{~s}^{-1}$ (e.g., Sharp et al., 1981; Seki et al., 1998; Sauvaud et al., 2004). The distribution of $V_{\| X}$, measured in the PSBL, also has a maximum corresponding to velocities below $100 \mathrm{~km} \mathrm{~s}^{-1}$ and a long tail to higher values of $V_{\| X}$. This tail is provided by samples observed in the lobe side of the PSBL where ion beams move only in one direction (e.g., Takahashi and Hones, 1988). Deeper in the PSBL the presence of counterstreaming ion beams immediately decreases the ion bulk velocity up to zero values. And finally the presence of cold ion population in the PSBL additionally decreases the values of $V_{\| X}$.

The same situation is observed in the outer PS region. Since the ion velocity distribution function is almost isotropic there, even the presence of parallel ion flows mov-

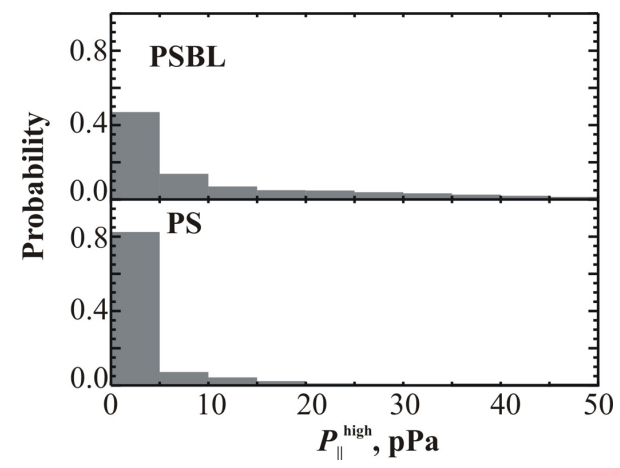

Fig. 7. Histograms of the distribution of the observation probability of the ion parallel dynamic pressure $P_{\|}^{\text {high }}$, calculated for the highenergy part of the spectrum ( $>2 \mathrm{keV}$ ) in the PSBL and the outer PS region.

ing earthward or tailward does not increase significantly the value of $V_{\| X}$. Thus, there is a significant overlap of $V_{\| X}$, measured in the PSBL and in the outer PS: approximately $98 \%$ of the outer PS samples and $\sim 70 \%$ of the PSBL samples are characterised by $V_{\|} \leq 400 \mathrm{~km} \mathrm{~s}^{1}$.

To separate the PSBL and the outer PS region we try to use the absolute value of the X-component of the parallel velocity calculated for the high energy part of the ion spectrum $(>2 \mathrm{keV}), V_{\| X}^{\text {high }}$ (see Fig. 8b). In the lobe region this parameter has zero value, since there are no ions with energies higher than $2 \mathrm{keV}$. The maximum of $V_{\| X}^{\text {high }}$ distribution, measured in the PSBL, corresponds to $V_{\| X}^{\text {high }}=400$ $600 \mathrm{~km} \mathrm{~s}^{-1}$, while in the outer PS region it corresponds to $V_{\| X}^{\text {high }} \leq 100 \mathrm{~km} \mathrm{~s}^{-1}$. The majority of samples in which $V_{\| X}^{\text {high }}<400 \mathrm{~km} \mathrm{~s}^{-1}$ belongs to the outer PS region (92\%), while samples with $V_{\| X}^{\text {high }} \geq 400 \mathrm{~km} \mathrm{~s}^{-1}$ most probably belong to the PSBL $(70 \%)$. Thus, an error in identification of the PSBL region by using the criterion $V_{\| X}^{\text {high }}>400 \mathrm{~km} \mathrm{~s}^{-1}$ is 0.3 and the error in identification of the outer PS region by using the criterion $V_{\| X}^{\text {high }}<400 \mathrm{~km} \mathrm{~s}^{-1}$ is only 0.08 . The use of higher $V_{\| X}^{\text {high }}$ values decreases the error of the outer PS identification and correspondingly increases the error for the PSBL separation.

Figure 9 shows a scatterplot of the ion density $N$ and the absolute value of the $\mathrm{X}$-component of the parallel velocity, $V_{\| X}$, calculated for the total energy range (a) and only for its high energy part $(>2 \mathrm{keV}, \mathrm{b})$. The PSBL samples are presented by blue dots and the outer PS samples - by red dots. We use a low density threshold for the moment calculation: if the density of the ions with energy $W>2 \mathrm{keV}$ is less than $0.01 \mathrm{~cm}^{-3}$ we put, for such samples, zero values of $N^{\text {high }}$ and $V_{\| X}^{\text {high }}$. Since in the lobe region ions with energies over $2 \mathrm{keV}$ are absent, the corresponding samples are not shown in the scatterplot. 

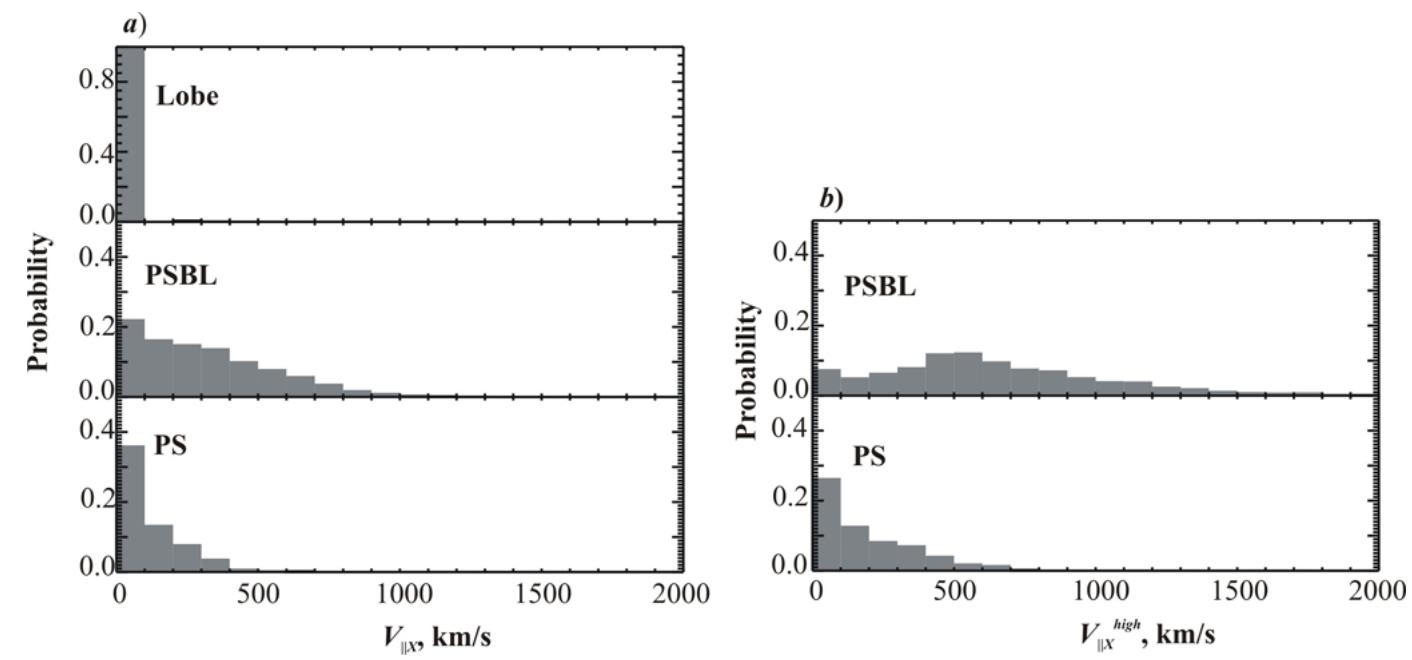

Fig. 8. A histogram of the distribution of the observation probability of the absolute value of the X-component of the ion parallel velocity calculated for the entire energy range, $V_{\| X}$ in the lobe, the PSBL and the outer PS region (a), and a histogram of the probability distribution of the $V_{\| X}^{\text {high }}$ calculated for the high-energy part of the spectrum $(>2 \mathrm{keV})$ in the PSBL and the outer PS region (b).

\section{a)}

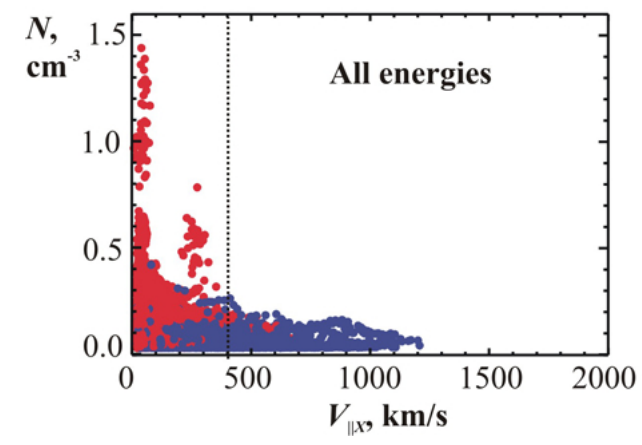

b)

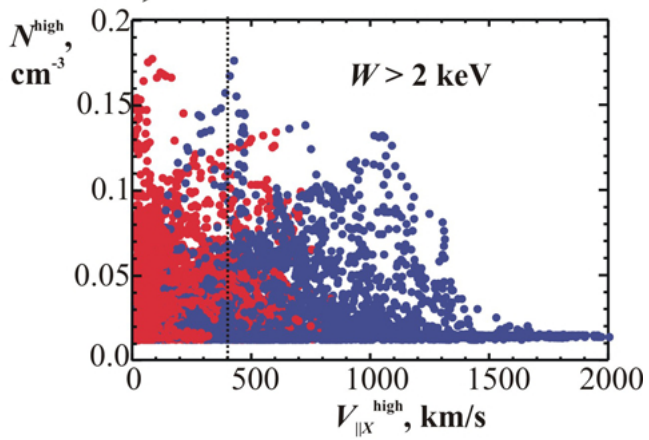

Fig. 9. (a) Scatterplot of the ion density $N$ versus the X-component of the parallel velocity $V_{\| X}$, calculated for the entire energy range; (b) the same moments, but calculated for the high-energy part of the spectrum ( $>2 \mathrm{keV})$. Data obtained in the PSBL are shown by blue dots and data measured in the outer PS region are displayed by red dots. Samples with very low densities $\left(<0.01 \mathrm{~cm}^{-3}\right)$ are not included.

The value of the ion density, calculated for the high energy part of the spectrum, $N^{\text {high }}$ is appropriate for the selection between the lobe region and the ion PSBL region, including the periods when there were no accelerated ion beams observed in the lobe-PS interface, since $N^{\text {high }}$ is zero in the lobe. But for separation between the PSBL and the outer PS region neither $N^{\text {high }}$ nor $N$ moments are appropriate. It is seen from Fig. 9 that there is a significant overlap in the values of the ion density $N$ and $N^{\text {high }}$, measured in both regions. Thus, only $V_{\| X}^{\text {high }}$ provides the best results in separation between the PSBL and the outer PS regions.

\section{Discussion}

In this paper, we analysed 100 crossings of the lobe-PSBLPS regions by Cluster spacecraft made in different periods of magnetotail activity. The selection of the events was random. Our study was based on Cluster observations in the magnetotail at $X \leq-15 R_{\mathrm{E}}$ during the period August-October of 2001. By visual inspection of the ion velocity distribution functions observed in these crossings we classified 32 events as intervals of the lobe-quasi-static PS crossings since in such events no field-aligned ion beams were observed. In 34 intervals of the lobe-PSBL-PS crossings Type-I ion beams were registered in the PSBL, and in the same number of events Type-II ion beams were detected in the PSBL.

The statistical analysis of the plasma parameters observed in these events was aimed to demonstrate that one should be very careful while performing PSBL identification by using ion moments without visual inspection of the velocity distribution functions. We have checked different ion moments: the density and the parallel velocity, calculated both for the 
Table 1. A percentage of the lobe, PSBL and outer PS samples for particular ranges of plasma $\beta$ values.

\begin{tabular}{llll}
\hline & Lobe & PSBL & Outer PS \\
\hline$\beta<0.01$ & $91 \%$ & $7 \%$ & $2 \%$ \\
$0.01 \leq \beta<0.1$ & $20 \%$ & $54 \%$ & $26 \%$ \\
$0.1 \leq \beta<0.2$ & $3 \%$ & $56 \%$ & $41 \%$ \\
$0.2 \leq \beta<0.5$ & $1 \%$ & $38 \%$ & $61 \%$ \\
$0.5 \leq \beta<1.0$ & $1 \%$ & $37 \%$ & $62 \%$ \\
$1.0 \leq \beta<5.0$ & $0 \%$ & $17 \%$ & $83 \%$ \\
$5.0 \leq \beta<10.0$ & $0 \%$ & $0 \%$ & $100 \%$ \\
\hline
\end{tabular}

total energy range and for the high energy part of the spectrum, and their combinations like the plasma $\beta$ and the parallel dynamic pressure. We revealed that the use of a combination of moments including the ion density and velocity (or temperature) provides the worst results in PSBL separation especially from the outer PS region. The problem is that during different periods of magnetotail activity the ion density, the temperature and the flow velocity in the PSBL and in the outer PS may vary in wide range of values.

In Table 1 we demonstrate how values of the plasma $\beta$ from a particular $\beta$ range are distributed among the lobe, the PSBL and the outer PS samples. For this purpose, we calculate the probability to observe $\beta$ values from a particular $\beta$ range in these three regions as: $P_{[\beta] \text { lobe }}=N_{[\beta] \text { lobe }} / N_{[\beta]}$; $P_{[\beta] \mathrm{PSBL}}=N_{[\beta] \mathrm{PSBL}} / N_{[\beta]}$ and $P_{[\beta] \mathrm{PS}}=N_{[\beta] \mathrm{PS}} / N_{[\beta]}$. Here, $N_{[\beta] \text { lobe }}, N_{[\beta] \text { PSBL }}$ and $N_{[\beta] \text { PS }}$ represent the number of samples belonging to the lobe, the PSBL and the outer PS regions, respectively, which are characterised by $\beta$ values from the given $\beta$ range. $N_{[\beta]}$ is the total number of samples which are characterised by $\beta$ values from the given $\beta$ range.

The data presented in Table 1 and in Fig. 5 demonstrate that the lobe region could be distinguished rather well using $\beta$ values. Indeed, if the condition $\beta<0.01$ is used, $91 \%$ of the searched samples will belong to the lobe region (see Table 1). However, one should be aware of a possible 35\% loss of the lobe samples which are characterised by higher $\beta$ values, since the observation probability of $\beta<0.01$ in the lobe region is only $\sim 0.65$ (see Fig. 5 ). The condition of absence of high-energy ( $>2 \mathrm{keV}$ ) ions: $N^{\text {high }}<0.01 \mathrm{~cm}^{-3}$ provides the best result when separating the lobe region from the PSBL or the outer PS region. In our database it allows us to identify $99 \%$ of the lobe samples.

Separation between the PSBL and the outer PS regions according to the $\beta$ parameter is vague. For example, only $54 \%$ of the PSBL samples correspond to the $\beta$ range $0.01-$ 0.1 and the other ones have smaller or larger $\beta$ values (see Table 1). So if one uses this range of $\beta$ then approximately half of the PSBL data will be missed. Moreover, $46 \%$ of the samples having $\beta$ values from this range belong to the lobe and the outer PS regions.
PSBL intervals with field-aligned ion beams could be selected from the adjacent lobe and the outer PS regions by using the values of the $\mathrm{X}$-component of the ion parallel velocity $V_{\| X}^{\text {high }}$, calculated for the high-energy part of the spectrum ( $>2 \mathrm{keV}$ ). The condition: $V_{\| X}^{\text {high }} \geq 400 \mathrm{~km} \mathrm{~s}^{-1}$ allows identification of $70 \%$ of the PSBL samples. The usage of $V_{\| X}^{\text {high }}$ is more helpful in the identification of the lobe side of the PSBL, where the ion beams are moving only in one direction. Figure 10a presents a scatterplot of $N^{\text {high }}$ versus $V_{\| X}^{\text {high }}$, measured in the lobe side of the PSBL (blue dots) and in the outer PS region (red dots). It is seen that in the high-latitude part of the PSBL, where only one-direction ion beams are observed, the values of $V_{\| X}^{\text {high }}$ are statistically higher than in the other part of the PSBL and especially in the outer PS. Thus, the overlap with the outer PS samples is significantly smaller. For example, approximately $94 \%$ of the high-latitude samples have $V_{\| X}^{\text {high }} \geq 600 \mathrm{~km} \mathrm{~s}^{-1}$ values and $\sim 98 \%$ of the outer PS samples have values of $V_{\| X}^{\text {high }}<600 \mathrm{~km} \mathrm{~s}^{-1}$.

The separation of the inner part of the PSBL, where counterstreaming ion beams are observed, from the outer PS region is the most difficult task. And this problem does not depend on the type of the ion beam observed in the PSBL. Figure $10 \mathrm{~b}$ shows a scatterplot of $N^{\text {high }}$ versus $V_{\| X}^{\text {high }}$, measured in these two regions. The values of $V_{\| X}^{\text {high }}$ decrease statistically in the inner PSBL due to the presence of counterstreaming ion beams. Thus, $80 \%$ of the outer PS samples have $V_{\| X}^{\text {high }}<300 \mathrm{~km} \mathrm{~s}^{-1}$ and only $74 \%$ of the inner PSBL samples have $V_{\| X}^{\text {high }} \geq 300 \mathrm{~km} \mathrm{~s}^{-1}$. So that an error of $\sim 0.3$ is unavoidable when separating the inner part of PSBL from the outer PS region.

Finally, we may conclude that for the identification of the PSBL or the outer PS intervals the use of the plasma $\beta$ alone leads to large errors. For example, the use of the criterion $\beta<0.1$ for the search of PSBL samples results in $26 \%$ contamination by outer PS samples and $20 \%$ contamination by lobe samples (see Table 1). Moreover, in our database $\sim 40 \%$ of the PSBL samples are characterised by $\beta \geq 0.1$. Thus, the use of the $\beta<0.1$ criterion leads to the loss of these samples. Baumjohann et al. (1988) additionally used a criterion based on the value of the photoelectron flux, which may significantly improve the results of PSBL identification. Unfortunately, we cannot repeat their study with Cluster data.

The use of the plasma $\beta$ for the distinction between the PSBL and the outer PS intervals provides worse results. For example, the condition $\beta<0.5$, which was suggested by Angelopoulos et al. (1994) for the outer PS, actually corresponds to $\sim 82 \%$ of the outer PS samples from our database. However, approximately $94 \%$ of the PSBL samples from our database are characterised by $\beta<0.5$ as well. Thus, if one uses the plasma $\beta$ for the distinction between the PSBL and the outer PS regions, at least the additional criterion based on the value of the ion bulk velocity should be used. 

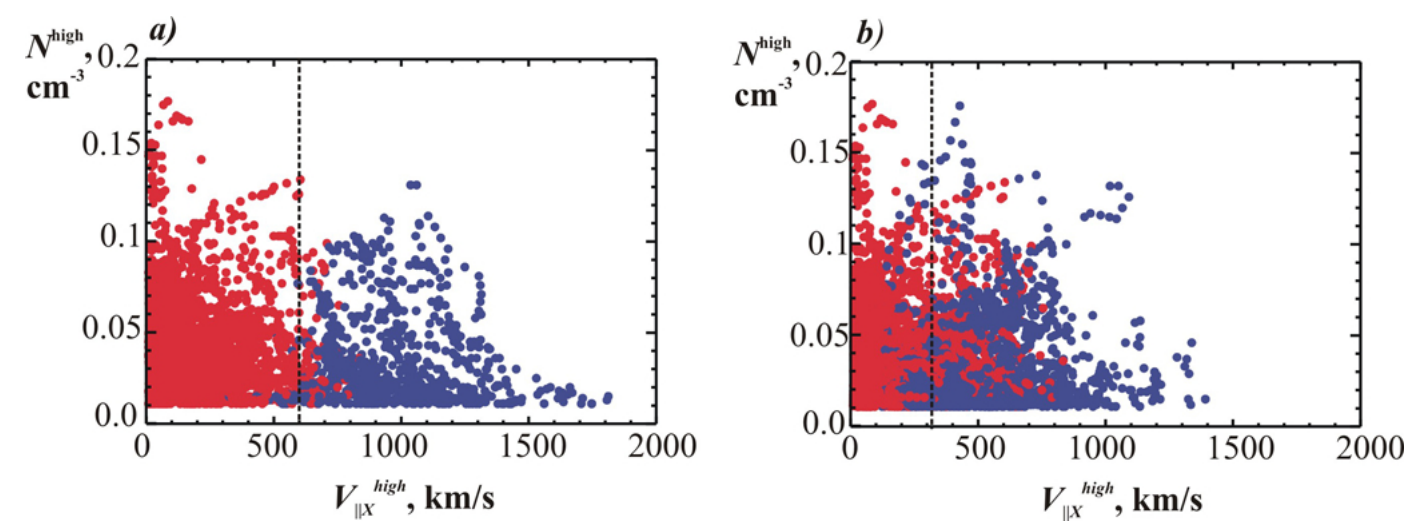

Fig. 10. (a) A scatterplot of the ion density $N^{\text {high }}$ versus the X-component of the parallel velocity $V_{\| X}^{\text {high }}$, calculated for the high-energy part of the spectrum in the lobe side of the PSBL (blue dots) and in the outer PS (red dots); (b) a scatterplot of the same moments, measured in the inner part of the PSBL, where counterstreaming ion beams are observed (blue dots) and in the outer PS (red dots). Samples with very low densities $\left(<0.01 \mathrm{~cm}^{-3}\right)$ are excluded.

\section{Conclusions}

In this paper we attempt to identify ion moments, which can be used for automatic moment based search of the PSBL region while doing statistical analysis of large volume of data. We demonstrate that the most difficult problem is related to the separation between the PSBL and the outer PS region, and we show the disadvantages of the use of ion moments and especially of their combination like the plasma $\beta$ for this task. The problem is that no clear boundary exists between the PSBL and the outer PS. Ion velocity distribution function gradually transforms from counterstreaming one inside the PSBL to a more isotropic distribution in which similar values of phase density are detected both in the field-aligned angular sectors and in the sectors corresponding to the perpendicular directions. Thus, during periods when no parallel flows moving in one particular direction are observed in the PSBL and the outer PS, the value of the ion bulk velocity in both regions is rather small.

The lobe-PS interface could be roughly classified in three states: (i) Type-I (energy-collimated) ion beams are propagating along magnetic field lines; (ii) Type-II ion beams, which are more energetic and are characterised by larger parallel temperature, are observed at the lobeward edge of the PSBL (Grigorenko et al., 2009) and (iii), the so-called quasistatic PS boundary, when no field-aligned ion beams are observed in the lobe-PS interface (Angelopoulos et al., 1993). In the last case this region is similar to the outer PS, which is adjacent to the PSBL. From our point of view, it is impossible to separate them. We use the common term "outer PS" for both regions.

Regardless of the presence of field-aligned ion beams in the lobe-PS interface, the lobe region could be separated from the lobe side of the ion PSBL or from the outer PS by the use of the following criteria: ion density calculated for the high-energy part of the spectrum $(>2 \mathrm{keV})$
$N^{\text {high }}<0.01 \mathrm{~cm}^{-3}$ (in our database $99 \%$ of the samples with such values of $N^{\text {high }}$ belong to the lobe region) or, but with a larger error $-\beta<0.01$ (in our database $66 \%$ of the lobe samples are characterised by these $\beta$ values).

The lobe side of the ion PSBL region during periods of Type-I or Type-II ion beam propagation could be separated from the inner PSBL and the outer PS regions by using the value of the $\mathrm{X}$-component of the ion parallel velocity calculated for the high-energy part of the spectrum $(>2 \mathrm{keV})$, $V_{\| X}^{\text {high }} \geq 600 \mathrm{~km} \mathrm{~s}^{-1}$. Almost $94 \%$ of the samples belonging to the high-latitude part of the PSBL satisfy this criterion.

The most difficult task is to separate the PSBL, especially its inner part, from the outer PS region. The condition $V_{\| X}^{\text {high }} \geq 400 \mathrm{~km} \mathrm{~s}^{-1}$ allows identification of $70 \%$ of the PSBL samples. The use of the plasma $\beta$ provides worse results. For example, $\beta$ values within the range $0.1-0.2$ are observed both in the PSBL and in the outer PS with approximately equal probability. The criterion $0.01 \leq \beta<0.1$ allows identification of only $54 \%$ of PSBL samples. The other searched samples belong either to the lobe or to the outer PS regions.

Thus, reasonable results could provide only momentbased search for PSBL regions containing field-aligned ion beams streaming in one direction. For their identification the absolute value of $V_{\| X}^{\text {high }}$ can be used together with the condition $N^{\text {high }} \geq 0.01 \mathrm{~cm}^{-3}$ in order to exclude the lobe region and to be sure in the reliability of the ion moment calculation. In order to separate the PSBL from the outer PS region, only a visual analysis of the ion velocity distribution functions allows avoiding significant errors. 
Acknowledgements. The authors thank the Cluster CIS, PEACE, FGM and CAA teams for providing the data. This work was supported by RFBR grants Nr. 10-02-00135; 10-02-93114, grant of Leading Scientific Schools HIII-623.2012.2 and grant DID 02/8 of the Bulgarian National Science Fund.

Topical Editor I. Daglis thanks two anonymous referees for their help in evaluating this paper.

\section{References}

Angelopoulos, V., Baumjohann, W., Kennel, C. F., Coroniti, F. V., Kivelson, M. G., Pellat, R., Walker, R. J., Lühr, H., and Paschmann, G.: Bursty bulk flows in the inner central plasma sheet, J. Geophys. Res., 97, 4027-4039, 1992.

Angelopoulos, V., Kennel, C. F., Coroniti, F. V., Feldman, W. C., Gosling, J. T., Kivelson, M. G., Walker, R. J., and Russell, C. T.: Observations of a quasi-static plasma sheet boundary, Geophys. Res. Lett., 20, 2813-2816, 1993.

Angelopoulos, V., Kennel, C. F., Coroniti, F. V., Pellat, R., Kivelson, M. G., Walker, R. J., Russell, C. T., Baumjohann, W., Feldman, W. C., and Gosling, J. T.: Statistical characteristics of bursty bulk flow events, J. Geophys. Res., 99, 21257-21280, 1994.

Balogh, A., Carr, C. M., Acuña, M. H., Dunlop, M. W., Beek, T. J., Brown, P., Fornacon, K.-H., Georgescu, E., Glassmeier, K.H., Harris, J., Musmann, G., Oddy, T., and Schwingenschuh, K.: The Cluster Magnetic Field Investigation: overview of in-flight performance and initial results, Ann. Geophys., 19, 1207-1217, doi:10.5194/angeo-19-1207-2001, 2001.

Baumjohann, W., Paschmann, G., Sckopke, N., Cattell, C. A., and Carlson, C. W.: Average ion moments in the plasma sheet boundary layer, J. Geophys. Res., 93, 11507, doi:10.1029/JA093iA10p11507, 1988.

Baumjohann, W., Paschmann, G., and Cattell, C. A.: Average plasma properties in the central plasma sheet, J. Geophys. Res., 94, 6597, doi:10.1029/JA094iA06p06597, 1989.

DeCoster, R. J. and Frank, L. A.: Observations pertaining to the dynamics of the plasma sheet, J. Geophys. Res., 84, 5099-5121, 1979.

Eastman, T. E., Frank, L. A., Peterson, W. K., and Lennartsson, W.: The plasma sheet boundary layer, J. Geophys. Res., 89, 15531572, 1984.

Forbes, T. G., Hones Jr., E. W., Bame, S. J., Asbridge, J. R., Paschmann, J., Sckopke, N., and Russell, C. T.: Evidence for the tailward retreat of a magnetic neutral line in the magnetotail during substorm recovery, Geophys. Res. Lett., 8, 261, doi:10.1029/GL008i003p00261, 1981.

Fujimoto, M., Nagai, T., Yokokawa, N., Yamade, Y., Mukai, T., Saito, Y., and Kokubun, S.: Tailward electrons at the lobe-plasma sheet interface detected upon dipolarizations, J. Geophys. Res., 106, 21255-21262, 2001.

Grigorenko, E. and Koleva, R.: Variability of discrete plasma structures in the lobe-plasma sheet interface, Compt. Rend. Acad. Bulg. Sci., 62, 1449-1456, 2009.

Grigorenko, E. E., Sauvaud, J.-A., and Zelenyi, L. M.: SpatialTemporal characteristics of ion beamlets in the plasma sheet boundary layer of magnetotail, J. Geophys. Res., 112, A05218, doi:10.1029/2006JA011986, 2007.

Grigorenko, E. E., Hoshino, M., Hirai, M., Mukai, T., and Zelenyi, L. M.: "Geography" of ion acceleration in the magnetotail: X- line versus current sheet effects, J. Geophys. Res., 114, A03203, doi:10.1029/2008JA013811, 2009.

Grigorenko, E. E., Burinskaya, T. M., Shevelev, M., Sauvaud, J.-A., and Zelenyi, L. M.: Large-scale fluctuations of PSBL magnetic flux tubes induced by the field-aligned motion of highly accelerated ions, Ann. Geophys., 28, 1273-1288, doi:10.5194/angeo28-1273-2010, 2010.

Grigorenko, E. E., Zelenyi, L. M., Dolgonosov, M. S., Artemiev, A. V., Owen, C. J., Sauvaud, J.-A., Hoshino, M., and Hirai, M.: Nonadiabatic ion acceleration in the Earth magnetotail and its various manifestations in the Plasma Sheet Boundary Layer, Space Sci. Rev., 164, 133-181, 2012.

Mukai, T., Hirahara, M., Machida, S., Saito, Y., Terasawa, T., and Nishida, A.: Geotail observations of cold ion streams in the medium distance magnetotail lobe in the course of a substorm, Geophys. Res. Lett., 21, 1023-1026, 1994.

Nagai, T. and Machida, S.: Magnetic reconnection in the near-Earth magnetotail, New Perspectives on the Earth's Magneottail, Geophys. Monogr. Ser., 105, edited by: Nishida, A., Baker, D. N., and Cowley, S. W. H., 211-224, AGU, Washington, D.C., 1998.

Nagai, T., Shinohara, I., Fujimoto, M., Hoshino, M., Saito, Y., Machida, S., and Mukai, T.: Geotail observations of the Hall current system: evidence of magnetic reconnection in the magnetotail, J. Geophys. Res., 106, 25929-25949, 2001.

Nakamura, M., Paschmann, G., Baumjohann, W., and Sckopke, N.: Ion distributions and flows in and near the neutral sheet, J. Geophys. Res., 96, 5631-5649, 1991.

Nakamura, R., Baumjohann, W., Nagai, T., Fujimoto, M., Mukai, T., Klecker, B., Treumann, R., Balogh, A., Rème, H., Sauvaud, J.-A., Kistler, L., Mouikis, C., Owen, C. J., Fazakerley, A. N., Dewhurst, J. P., and Bogdanova, Y.: Flow shear near the boundary of the plasma sheet observed by Cluster and Geotail, J. Geophys Res., 109, A05204, doi:10.1029/2003JA010174, 2004.

Owen, C. J., Fazakerley, A. N., Carter, P. J., Coates, A. J., Krauklis, I. C., Szita, S., Taylor, M. G. G. T., Travnicek, P., Watson, G., Wilson, R. J., Balogh, A., and Dunlop, M. W.: Cluster PEACE observations of electrons during magnetospheric flux transfer events, Ann. Geophys., 19, 1509-1522, doi:10.5194/angeo-191509-2001, 2001.

Parks, G., Chen, L. J., McCarthy, M., Larson, D., Lin, R. P., Phan, T., Reme, H., and Sanderson, T.: New observations of ion beams in the plasma sheet boundary layer, Geophys. Res. Lett., 25, 3285-3288, 1998

Petrukovich, A. A., Baumjohann, W., Nakamura, R., Schödel, R., and Mukai, T.: Are earthward bursty bulk flows convective or field-aligned?, J. Geophys. Res., 106, 21211-21215, 2001.

Raj, A., Phan, R., Lin, R. P., and Angelopoulos, V.: Wind survey of high-speed bulk flows and field-aligned beams in the near-Earth plasma sheet, J. Geophys. Res., 107, 1419, doi:10.1029/2001JA007547, 2002.

Rème, H., Aoustin, C., Bosqued, J. M., Dandouras, I., Lavraud, B., Sauvaud, J. A., Barthe, A., Bouyssou, J., Camus, Th., Coeur-Joly, O., Cros, A., Cuvilo, J., Ducay, F., Garbarowitz, Y., Medale, J. L., Penou, E., Perrier, H., Romefort, D., Rouzaud, J., Vallat, C., Alcaydé, D., Jacquey, C., Mazelle, C., d'Uston, C., Möbius, E., Kistler, L. M., Crocker, K., Granoff, M., Mouikis, C., Popecki, M., Vosbury, M., Klecker, B., Hovestadt, D., Kucharek, H., Kuenneth, E., Paschmann, G., Scholer, M., Sckopke, N., Seidenschwang, E., Carlson, C. W., Curtis, D. W., Ingraham, C., Lin, R. 
P., McFadden, J. P., Parks, G. K., Phan, T., Formisano, V., Amata, E., Bavassano-Cattaneo, M. B., Baldetti, P., Bruno, R., Chionchio, G., Di Lellis, A., Marcucci, M. F., Pallocchia, G., Korth, A., Daly, P. W., Graeve, B., Rosenbauer, H., Vasyliunas, V., McCarthy, M., Wilber, M., Eliasson, L., Lundin, R., Olsen, S., Shelley, E. G., Fuselier, S., Ghielmetti, A. G., Lennartsson, W., Escoubet, C. P., Balsiger, H., Friedel, R., Cao, J.-B., Kovrazhkin, R. A., Papamastorakis, I., Pellat, R., Scudder, J., and Sonnerup, B.: First multispacecraft ion measurements in and near the Earth's magnetosphere with the identical Cluster ion spectrometry (CIS) experiment, Ann. Geophys., 19, 1303-1354, doi:10.5194/angeo19-1303-2001, 2001.

Sauvaud, J.-A., Louarn, P., Fruit, G., Stenuit, H., Vallat, C., Dandouras, J., Rème, H., André, M., Balogh, A., Dunlop, M., Kistler, L., Möbius, E., Mouikis, C., Klecker, B., Parks, G. K., McFadden, J., Carlson, C., Marcucci, F., Pallocchia, G., Lundin, R., Korth, A., and McCarthy, M.: Case studies of the dynamics of ionospheric ions in the Earth's magnetotail, J. Geophys. Res., 109, A01212, doi:10.1029/2003JA009996, 2004.
Seki, K., Hirahara, M., Terasawa, T., Mukai, T., Saito, Y., Machida, S., Yamamoto, T., and Kokubun, S.: Statistical properties and possible supply mechanisms of tailward cold $\mathrm{O}^{+}$beams in the lobe/mantle regions, J. Geophys. Res., 103, 4477-4493, 1998.

Sharp, R. D., Carr, D. L., Peterson, W. K., and Shelley, E. G.: Ion Streams in the Magnetotail, J. Geophys. Res., 86, 4639-4648, 1981.

Takahashi, K. and Hones, E. W.: ISEE 1 and 2 observations of ion distributions at the plasma sheet-tail lobe boundary, J. Geophys. Res., 93, 8558-8582, 1988. 